\title{
A narrative review of hyperthermic intrathoracic chemotherapy for advanced lung cancer
}

\author{
Kimberly Song, Raja M. Flores \\ Department of Thoracic Surgery, Icahn School of Medicine at Mount Sinai, Mount Sinai Health System, New York, USA \\ Contributions: (I) Conception and design: Both authors; (II) Administrative support: Both authors; (III) Provision of study materials or patients: \\ Both authors; (IV) Collection and assembly of data: Both authors; (V) Data analysis and interpretation: Both authors; (VI) Manuscript writing: Both \\ authors; (VII) Final approval of manuscript: Both authors. \\ Correspondence to: Raja M. Flores. Department of Thoracic Surgery, Icahn School of Medicine at Mount Sinai, Mount Sinai Health System, New \\ York, USA. Email: raja.flores@mountsinai.org.
}

\begin{abstract}
The traditional treatment of stage IV lung cancer is predominantly supportive or palliative. No current standardized guidelines promote the use of hyperthermic intrathoracic chemotherapy (HITHOC) in the treatment of advanced lung cancer with pleural involvement. Several small studies have examined the safety and utilization of HITHOC for this population, though the data is extremely limited. A review of the literature is presented in accordance with the Narrative Review checklist. The MEDLINE electronic database was searched for articles published in English from January 1999 - August 2020 using relevant keywords such as "hyperthermic intrathoracic chemotherapy", "hyperthermic intrapleural chemotherapy" and "HITHOC". This was supplemented by review and hand search of the reference lists. While data suggest a potential though controversial role for HITHOC for certain intrathoracic tumors such as malignant pleural mesothelioma and thymoma, there is insufficient evidence to confidently promote a role for hyperthermic intrathoracic chemotherapy in the treatment of advanced lung cancers. Existing studies are small, nonrandomized, and prone to bias. Hyperthermic intrathoracic chemotherapy is not a standardized treatment for advanced lung cancer, and is characterized by potentially serious side effects with little clinical benefit. Recent developments in targeted therapy and immunotherapy are unlikely to leave room for the development of large randomized controlled trials.
\end{abstract}

Keywords: Hyperthermic intrathoracic chemotherapy (HITHOC); malignant pleural effusion; advanced lung cancer; non-small cell lung cancer (NSCLC)

Submitted Sep 19, 2020. Accepted for publication Mar 23, 2021.

doi: $10.21037 / \mathrm{atm}-20-6514$

View this article at: http://dx.doi.org/10.21037/atm-20-6514

\section{Introduction}

Hyperthermic intrathoracic chemotherapy (HITHOC) involves the local administration of cytotoxic therapy in the pleural space and is a relatively uncommon treatment modality for pleural disease and malignant effusions. Though HITHOC has been used for intrathoracic tumors such as thymoma and malignant pleural mesothelioma, albeit controversially, its application in advanced primary lung cancer has been limited; traditional treatment of pleural involvement in stage IV lung cancer is predominantly supportive or palliative. Several small studies have examined the safety and utilization of HITHOC for this patient population; here we review the available literature to examine its role in the current treatment of these patients, and present this information in accordance with the Narrative Review reporting checklist (1) (available at http://dx.doi.org/10.21037/atm-20-6514).

\section{Methods}

The MEDLINE electronic database was searched for 
articles from January 1999 - August 2020 using the keywords "hyperthermic intrathoracic chemotherapy", "hyperthermic intrapleural chemotherapy" and "HITHOC", supplemented by review of the reference lists from these articles. The majority of studies and reports were published after the year 2013, and a total of 84 papers written in English were identified. Those focused primarily on the treatment of metastatic disease from extrathoracic primaries were excluded. Sixty-eight papers meeting these criteria, including those discussing the use of HITHOC for thymoma or malignant pleural mesothelioma rather than advanced lung cancer, were identified and reviewed for discussion. Papers primarily focused on non-lung primary or intraperitoneal disease were excluded.

\section{Background}

The groundwork for modern intraoperative chemotherapy began as far back as the 1970s with early concepts of hyperthermic intraperitoneal chemotherapy (HIPEC) (1). This procedure has become increasingly common for intraabdominal tumors and malignant ascites (2). The efficacy of HITHOC is substantially less well described or supported in the literature, with several small reports asserting its safety for certain patient populations; most currently available literature originates from Asia (3).

The rationale for intraperitoneal or intrapleural chemotherapy focuses on the concentrated local delivery of cytotoxic agents with reduced systemic absorption and toxicity. Research has shown that intrapleural levels of cisplatin and mitomycin after pleurectomy/decortication maintain lower systemic levels than in pleural fluid (4) while tumor cells from the malignant effusions of patients with primary lung adenocarcinoma show a significantly increased rate of apoptosis 24 hours after intrapleural exposure to cisplatin (5). While certain intraabdominal tumors treated with HIPEC have shown clear sensitivity to hyperthermia (6), other evidence regarding clinically relevant thermosensitivity for intrathoracic malignancies has been less clear (7). Most published HITHOC techniques employ varying degrees of hyperthermia, and Zhang et al. have demonstrated effectiveness of hyperthermia with cisplatin in downregulating epidermal growth factor receptor (EGFR) proteins in EGFR kinase mutation positive lung cancers, with subsequently increased apoptotic response (8).

In the most common general techniques, a pleurectomy and/or debulking is first performed under general anesthesia, followed by consistent instillation of a cisplatinbased fluid mixture at a temperature range of $42-45{ }^{\circ} \mathrm{C}$ for 30-90 $\min (9-12)$. Attention to core body temperature maintenance is critical and patients require careful postoperative monitoring to address potential side effects. Although most published reports involve intraoperative administration of HITHOC after surgical debulking, bedside approaches have also been described (13).

\section{Current use of HITHOC for metastatic disease}

Case reports describing HITHOC for metastatic disease from extrathoracic sites have primarily focused on pseudomyxoma peritonei and ovarian cancer. The former is a tumor that produces mucinous ascites in the peritoneal cavity that can rarely spread to the pleural space. While it most frequently arises from appendiceal cancer, it can be caused by other types of tumors including ovarian cancer. HIPEC with cytoreduction has demonstrated prolonged survival for pseudomyxoma peritonei (14). Isolated reports of debulking and HITHOC have been described with some success for both pseudomyxoma peritonei and ovarian carcinoma (15-19).

\section{Application in malignant pleural mesothelioma}

HITHOC is commonly used to reduce microscopic disease and one of its more established applications, though still controversial, is for residual tumor burden in malignant pleural mesothelioma (MPM). A small study by Migliore et al. showed low morbidity and no perioperative mortality when intrapleural cisplatin was administered to young patients with good performance status, early disease, and predominantly epithelioid histology (12) and survival improvements have been demonstrated in select populations of patients (10).

The preferred regimen for HITHOC in MPM is unclear. In vitro assessment of the response of mesothelioma cell lines to varying combinations of cisplatin, gemcitabine, and pemetrexed with and without hyperthermia have indicated increased responses to multidrug therapy, with less benefit from isolated or added hyperthermia up to $45^{\circ} \mathrm{C}(7)$. In a report of their ten-year experience with 49 patients, Ambrogi et al. employed a combination of cisplatin and epirubicin during HITHOC for epithelioid or biphasic MPM and demonstrated good outcomes using a surgical model focused on preserving lung parenchyma and diaphragmatic function (20). However, no randomized 
controlled trials have been published and it remains uncertain which population of patients would benefit over more standard treatment patterns.

\section{Application in pleural manifestations of thymoma}

Thymomas, the most common anterior mediastinal tumors, are often indolent though malignant neoplasms that can be accompanied by neuromuscular paraneoplastic syndromes, the most common being myasthenia gravis (21). These tumors are best treated by complete resection but require long-term follow up after surgery due to recurrence rates as high as $30-50 \%$ after initial surgery. Pleural disease remains the most common site at about $50 \%$ of recurrences (22). There is limited evidence from small studies to suggest a possible role for intrapleural cisplatin in stage IVa disease with known pleural involvement at the time of initial resection, with reports of patients having good survival with low recurrence rates $(23,24)$.

A small study examining the role of HITHOC for pleural based recurrences of thymoma in patients varying widely in their initial Masaoka stage, WHO classification, concurrent diagnosis of myasthenia gravis, and neoadjuvant or adjuvant treatment strategy found HITHOC to be safe and feasible, with most complications involving prolonged air leak $(85 \%)$ or bleeding $(23 \%)$. During a mean follow up period of 64.6 months, 4 patients developed pleural relapse and 1 developed progression of disease (25). As with MPM, clear guidelines for the use of HITHOC in thymic disease are lacking.

\section{Current role in advanced primary lung cancer}

While the concurrent application of HITHOC has been successfully used for MPM and thymoma, very few studies exist examining the role of HITHOC for advanced primary lung malignancies and even fewer have further compared results by cancer subtype or molecular markers. Preliminary investigations aimed at suggesting safety and feasibility seem to have done so. A 2003 Japanese report of HITHOC in 5 patients with malignant effusion from non-small cell lung cancer (NSCLC) reported a median survival of 19 months with 4 patients still alive at the time of publication (26). The selection criteria in this study were strict-all patients were relatively young (mean age of 58), lacked N2 disease, and because the design of this study required pneumonectomy, all required good baseline performance status. It is impossible to distinguish the degree to which patient selection contributed to outcome, and the authors did not provide information on post-pneumonectomy quality of life. A more recent study in patients with advanced NSCLC reported a zero mortality rate during 9-35 months followup and greater freedom from effusion in those receiving HITHOC, but the study size was only 19 patients and there was significant heterogeneity in the adjuvant chemotherapy between groups (27). A recent systematic review involving 4 publications with a total of 21 patients receiving debulking + HITHOC for malignant effusion from NSCLC found a mean survival of 27 months, with $62 \%$ alive at 1 year and $28.5 \%$ alive at 2 years. Insufficient data was available in this study to perform a meta-analysis (3).

Studies aimed at determining HITHOC's clinical benefit have shown some frail success. A retrospective review of 23 patients with advanced adenocarcinoma undergoing surgical resection ranging from wedge to bilobectomy (but most commonly lobectomy, $56.5 \%$ ) followed by intrapleural cisplatin had improved overall survival compared to surgery alone, though no benefit in progression free survival was found (9). Results from this study are skewed by lack of randomization and stark differences between groups, most notably a bias toward later $\mathrm{T}$ and $\mathrm{N}$ stages in patients receiving surgery alone. In a small series of less than 20 patients, $63 \%$ with a primary lung malignancy, HITHOC and cisplatin-based systemic therapy administered after debulking via pleurectomy or resection was associated with improved survival at one year compared to debulking and systemic therapy only, and overall survival was improved in those with primary lung cancer. However, the controls in this analysis were purely historical (28). Zhou et al. published a meta-analysis of five studies showing that the addition of HITHOC to more traditional treatment modalities (systemic therapy or cytoreductive therapy) was associated with increased 1-year overall survival, increased disease-free survival, and improved Karnofsky performance status. Again, the included studies varied widely in treatment protocol and study arm design (29).

While concurrent chemoradiation is the foundation of treatment for advanced lung cancer, intraoperative chemotherapy could have a role in the symptomatic management of malignant effusion, as has been demonstrated for the management of malignant ascites (30). Though the most common surgical treatment options for pleural dissemination with associated effusion include palliative pleurodesis or indwelling catheter placement, the small study by Kimura et al. showed 
improved freedom from effusion (27) and further prospective investigation might be warranted. In a recent prospective randomized comparison of HITHOC $v s$. talc pleurodesis for malignant pleural effusion in NSCLC, the authors reported no difference in overall survival but demonstrated comparable safety and efficacy with their HITHOC technique (31). $\mathrm{Hu}$ et al. performed only thoracoscopic pleural biopsy with HITHOC for 54 patients with NSCLC and reported significant control of effusion in all patients over a median survival time of 21.7 months (32). No significant large scale data has been published demonstrating successful long-term control of malignant pleural effusion.

\section{Safety and side effects}

The most common surgical complications of HITHOC appear to be prolonged airleak, bleeding, and empyema $(25,33)$. A recent systematic review of 27 articles discussed a lack of perioperative or HITHOC-associated mortality in all included papers, though morbidity rates remain relatively high at 30\% (29). Van Sandick et al. reported a higher rate of complications in MPM patients treated with extrapleural pneumonectomy $(\mathrm{EPP})+$ radiation $(\mathrm{n}=15)$ vs. EPP or pleurectomy + HITHOC + radiation $(\mathrm{n}=20)$. The most common complications in the latter group were diaphragmatic rupture $(\mathrm{n}=5)$ and bronchopleural fistula $(n=4)$. This group also included two postoperative deaths and had a significantly shorter time to local recurrence (34).

More serious immediate intraoperative complications have also been reported. In their study of 20 patients with mesothelioma or Stage IVa thymoma, Kersecher et al. reported half of the patients in their study demonstrating significant increase in central venous pressure, increase in peak airway pressure, and hemodynamic changes requiring vasopressors upon initiation of HITHOC. One patient developed asystole requiring cardiopulmonary resuscitation, but recovered after surgery without neurologic deficit (35).

Bedside administration under local anesthesia may have fewer serious procedural morbidities but are likely to require repeated procedures and do not have the benefit of surgical debulking. In a 2016 retrospective study of over 300 patients receiving bedside intrapleural chemotherapy more than half of patients required 4-6 treatments, the need for which was determined by serial imaging. Approximately half had advanced primary lung cancer and $4.8 \%$ had malignant thymoma; the remainder were extrathoracic primaries. The most common side effect was pneumothorax or pain and no information regarding long term success of the procedure was described (13).

As in HIPEC, proponents of HITHOC hope that locally administered cytotoxic agents can be given at higher doses with limited systemic absorption and toxicity. Non-surgical morbidity is focused on the prevention of nephrotoxicity and renal failure associated with cisplatin, which is the mainstay of intrathoracic chemotherapy as in established systemic regimens. Low grade renal toxicity can occur in as many as $30 \%$ of patients with baseline normal kidney function and good performance status after cisplatin HITHOC (36). Typical renoprotective measures including resuscitative fluids and diuretics are given, and sodium thiosulfate may limit systemic absorption of platinumbased HITHOC (11). A recent study of 84 patients receiving intrapleural hyperthermic cisplatin + doxorubicin demonstrated that cytoprotection with perioperative fluid support was associated with fewer and less pronounced renal effects even when given in conjunction with higher concentrations of cisplatin (37).

\section{Summary}

Much needs to be done to further elucidate a potential role of intrapleural chemotherapy with or without hyperthermia for intrathoracic tumors. While HITHOC has demonstrated some weak benefit as an adjunct to cytoreduction for pleural dissemination of thymoma and malignant pleural mesothelioma, its utility for advanced primary lung cancers is quite poorly established and is not a generally recommended adjunct for the treatment of stage IV lung cancer. The available clinical data is scarce, heterogeneous, and lacking in large scale prospective randomized trials. Such trials are unlikely to be pursued in the near future given more recent advances in biomarkertargeted and immune therapy (38). The only clear conclusion that can currently be made is that there is no proven substantial benefit for HITHOC in these patients.

\section{Acknowledgments}

Funding: None.

\section{Footnote}

Provenance and Peer Review: This article was commissioned by the Guest Editor (Marcello Migliore) for the series "Hyperthermic Intraoperative Chemotherapy (HITHOC) in thoracic surgical oncology" published in Annals of 
Translational Medicine. The article has undergone external peer review.

Reporting Checklist: The authors have completed the Narrative Review reporting checklist. Available at http:// dx.doi.org/10.21037/atm-20-6514

Conflicts of Interest: Both authors have completed the ICMJE uniform disclosure form (available at http://dx.doi. org/10.21037/atm-20-6514). The series "Hyperthermic Intraoperative Chemotherapy (HITHOC) in thoracic surgical oncology" was commissioned by the editorial office without any funding or sponsorship. The authors have no other conflicts of interest to declare.

Ethical Statement: The authors are accountable for all aspects of the work in ensuring that questions related to the accuracy or integrity of any part of the work are appropriately investigated and resolved.

Open Access Statement: This is an Open Access article distributed in accordance with the Creative Commons Attribution-NonCommercial-NoDerivs 4.0 International License (CC BY-NC-ND 4.0), which permits the noncommercial replication and distribution of the article with the strict proviso that no changes or edits are made and the original work is properly cited (including links to both the formal publication through the relevant DOI and the license). See: https://creativecommons.org/licenses/by-nc-nd/4.0/.

\section{References}

1. Dedrick RL, Myers CE, Bungay PM, et al. Pharmacokinetic rationale for peritoneal drug administration in the treatment of ovarian cancer. Cancer Treat Rep 1978;62:1-11.

2. Bushati M, Rovers KP, Sommariva A, et al. The current practice of cytoreductive surgery and HIPEC for colorectal peritoneal metastases: Results of a worldwide webbased survey of the Peritoneal Surface Oncology Group International (PSOGI). Eur J Surg Oncol 2018;44:1942-8.

3. Migliore $M$, Nardini M. Does cytoreduction surgery and hyperthermic intrathoracic chemotherapy prolong survival in patients with N0-N1 nonsmall cell lung cancer and malignant pleural effusion? Eur Respir Rev 2019;28:190018.

4. Rusch VW, Niedzwiecki D, Tao Y, et al. Intrapleural cisplatin and mitomycin for malignant mesothelioma following pleurectomy: pharmacokinetic studies. J Clin Oncol 1992;10:1001-6.

5. Matsuzaki Y, Edagawa M, Shimizu T, et al. Intrapleural hyperthermic perfusion with chemotherapy increases apoptosis in malignant pleuritis. Ann Thorac Surg 2004;78:1769-72.

6. Barlogie B, Corry PM, Drewinko B. In vitro thermochemotherapy of human colon cancer cells with cisdichlorodiammineplatinum(II) and mitomycin C. Cancer Res 1980;40:1165-8.

7. Cameron RB, Hou D. Intraoperative hyperthermic chemotherapy perfusion for malignant pleural mesothelioma: an in vitro evaluation. J Thorac Cardiovasc Surg 2013;145:496-504.

8. Zhang $\mathrm{H}$, Zhan $\mathrm{C}, \mathrm{Ke}$ J, et al. EGFR kinase domain mutation positive lung cancers are sensitive to intrapleural perfusion with hyperthermic chemotherapy (IPHC) complete treatment. Oncotarget 2016;7:3367-78.

9. Yi E, Kim D, Cho S, Kim K, et al. Clinical outcomes of cytoreductive surgery combined with intrapleural perfusion of hyperthermic chemotherapy in advanced lung adenocarcinoma with pleural dissemination. J Thorac Dis 2016;8:1550-60.

10. Sugarbaker DJ, Gill RR, Yeap BY, et al. Hyperthermic intraoperative pleural cisplatin chemotherapy extends interval to recurrence and survival among low-risk patients with malignant pleural mesothelioma undergoing surgical macroscopic complete resection. J Thorac Cardiovasc Surg 2013;145:955-63.

11. Petrov R, Bakhos C, Abbas AE. Management of Malignant Lung Entrapment, the Oncothorax. Thorac Surg Clin 2018;28:81-90.

12. Migliore M, Calvo D, Criscione A, et al. Pleurectomy/ decortication and hyperthermic intrapleural chemotherapy for malignant pleural mesothelioma: initial experience. Future Oncol 2015;11:19-22.

13. Liu L, Zhang N, Min J, et al. Retrospective analysis on the safety of 5,759 times of bedside hyperthermic intraperitoneal or intra-pleural chemotherapy (HIPEC). Oncotarget 2016;7:21570-8.

14. Sugarbaker PH. Comprehensive management of peritoneal surface malignancy using cytoreductive surgery and perioperative intraperitoneal chemotherapy: the Washington Cancer Institute approach. Expert Opin Pharmacother 2009;10:1965-77.

15. Saladino E, Famulari C, La Monaca E, et al. Cytoreductive surgery plus bicavitary chemohyperthermia as treatment of pseudomixoma peritonei with pleural extension. A 
case report and review of the literature. Ann Ital Chir 2014;85:372-6.

16. Kawaguchi Y, Hanaoka J. Cytoreductive Surgery and Hyperthermic Chemotherapy for Intrathoracic Pseudomyxoma Peritonei. Ann Thorac Surg 2020;110:e541-e543.

17. Jun SY, Seok YK, Kato T, et al. Hyperthermic intrathoracic chemotherapy with cisplatin for ovarian cancer with pleural metastasis. Obstet Gynecol Sci 2017;60:308-13.

18. Stojiljkovic D, Nikolic S, Cvetkovic A, et al. Hyperthermic intrathoracic chemotherapy (HITHOC) in ovarian carcinoma - a propos of a case. J BUON 2018;23:153-5.

19. Dattatri R, Garg R, Ray MD. Cytoreductive onco-surgery with combined hyperthermic intraperitoneal chemotherapy and hyperthermic intrathoracic chemotherapy: Perioperative challenges. Saudi J Anaesth 2019;13:131-5.

20. Ambrogi MC, Bertoglio P, Aprile V, et al. Diaphragm and lung-preserving surgery with hyperthermic chemotherapy for malignant pleural mesothelioma: A 10-year experience. J Thorac Cardiovasc Surg 2018;155:1857-66.e2.

21. Evoli A, Lancaster E. Paraneoplastic disorders in thymoma patients. J Thorac Oncol 2014;9:S143-7.

22. Ruffini E, Filosso PL, Oliaro A. The role of surgery in recurrent thymic tumors. Thorac Surg Clin 2009;19:121-31.

23. Ried M, Potzger T, Braune N, et al. Cytoreductive surgery and hyperthermic intrathoracic chemotherapy perfusion for malignant pleural tumours: perioperative management and clinical experience. Eur J Cardiothorac Surg 2013;43:801-7.

24. Refaely Y, Simansky DA, Paley M, et al. Resection and perfusion thermochemotherapy: a new approach for the treatment of thymic malignancies with pleural spread. Ann Thorac Surg 2001;72:366-70.

25. Ambrogi MC, Korasidis S, Lucchi M, et al. Pleural recurrence of thymoma: surgical resection followed by hyperthermic intrathoracic perfusion chemotherapy†. Eur J Cardiothorac Surg 2016;49:321-6.

26. Shigemura N, Akashi A, Ohta M, et al. Combined surgery of intrapleural perfusion hyperthermic chemotherapy and panpleuropneumonectomy for lung cancer with advanced pleural spread: a pilot study. Interact Cardiovasc Thorac Surg 2003;2:671-5.

27. Kimura M, Tojo T, Naito H, et al. Effects of a simple intraoperative intrathoracic hyperthermotherapy for lung cancer with malignant pleural effusion or dissemination. Interact Cardiovasc Thorac Surg 2010;10:568-71.

28. Işı1k AF, Sanlı M, Yılmaz M, et al. Intrapleural hyperthermic perfusion chemotherapy in subjects with metastatic pleural malignancies. Respir Med 2013;107:762-7.

29. Zhou H, Wu W, Tang X, et al. Effect of hyperthermic intrathoracic chemotherapy (HITHOC) on the malignant pleural effusion: A systematic review and meta-analysis. Medicine (Baltimore) 2017;96:e5532.

30. Randle RW, Swett KR, Swords DS, et al. Efficacy of cytoreductive surgery with hyperthermic intraperitoneal chemotherapy in the management of malignant ascites. Ann Surg Oncol 2014;21:1474-9.

31. Kleontas A, Sioga A, Pandria N, et al. Clinical factors affecting the survival of patients diagnosed with non-small cell lung cancer and metastatic malignant pleural effusion, treated with hyperthermic intrathoracic chemotherapy or chemical talc pleurodesis: a monocentric, prospective, randomized trial. J Thorac Dis 2019;11:1788-98.

32. Hu $\mathrm{R}$, Jiang $\mathrm{H}, \mathrm{Li} \mathrm{H}$, et al. Intrapleural perfusion thermo-chemotherapy for pleural effusion caused by lung carcinoma under VATS. J Thorac Dis 2017;9:1317-21.

33. Yellin A, Simansky DA, Paley M, et al. Hyperthermic pleural perfusion with cisplatin: early clinical experience. Cancer 2001;92:2197-203.

34. van Sandick JW, Kappers I, Baas P, et al. Surgical treatment in the management of malignant pleural mesothelioma: a single institution's experience. Ann Surg Oncol 2008;15:1757-64.

35. Kerscher C, Ried M, Hofmann HS, et al. Anaesthetic management of cytoreductive surgery followed by hyperthermic intrathoracic chemotherapy perfusion. J Cardiothorac Surg 2014;9:125.

36. Sakaguchi H, Ishida H, Nitanda H, et al. Pharmacokinetic evaluation of intrapleural perfusion with hyperthermic chemotherapy using cisplatin in patients with malignant pleural effusion. Lung Cancer 2017;104:70-4.

37. Markowiak T, Kerner N, Neu R, et al. Adequate nephroprotection reduces renal complications after hyperthermic intrathoracic chemotherapy. J Surg Oncol 2019;120:1220-6.

38. Arbour KC, Riely GJ. Systemic Therapy for Locally Advanced and Metastatic Non-Small Cell Lung Cancer: A Review. JAMA 2019;322:764-74.

Cite this article as: Song K, Flores RM. A narrative review of hyperthermic intrathoracic chemotherapy for advanced lung cancer. Ann Transl Med 2021;9(11):958. doi: 10.21037/atm-206514 